\title{
Stop Control Strategy of Modular Multilevel Converter Based HVDC System
}

\author{
Chengjun Xia, Bingmei Jin, Junjun Liang, Meng Li \\ College of Electric Power, South China University of Technology, Guangzhou, China \\ Email: jinbingmei.123@163.com
}

Received July 2014

\begin{abstract}
The stop control strategy of modular multilevel converter based HVDC transmission system is proposed. This stop process is divided into stages of energy feedback and energy consumption. The DC voltage controller is coordinated to the used modules per phase when active power is transmitted prior to reactive power, so that the energy is fed back to the AC power grid connected to the converter station which uses the fixed dc voltage controller. In addition, in view of the different forms connected to the grid, specifically when the converter station supplies power for passive network, the passive converter station can take a certain auxiliary trigger strategy to make its maximum energy feedback to the grid. Finally, a simulation system of the MMC-HVDC system is constructed in Matlab/Simulink environment, and simulation results show that the proposed stop strategies are effective.
\end{abstract}

\section{Keywords}

Modular Multilevel Converter (MMC), Stop Control, Energy Feedback

\section{Introduction}

The normal shutdown of converter station refers to stop this DC line because of planned maintenance, change of system operation mode and so on and so forth, then the converter station equipments are required to ensure complete uncharged. According to the regulations of GB17702.1-1999, a capacitor which rated voltage is above $1 \mathrm{kV}$ should discharge its own to $75 \mathrm{~V}$ or less from the initial value within 10 minutes, you can use the appropriate control strategy to accelerate this discharge process. For traditional shutdown process, only directly latch the converter station after transmitted power is reduced to zero.

In the present work, the normal shutdown process is divided into energy feedback stage and energy consumption stage. The method in [1] can make the both converter stations feed about the same energy back to the AC electric grid, which improves the utilization of energy, and reduces the pressure level and power level of the discharge resistance. Another shutdown method with considering the sub-module redundancy is proposed in which situation MMC-HVDC systems is connected to passive network, which can further feed energy back to the grid than in [1], but converter station will consume a certain inductive reactive in the process of energy feedback [2]. In general this is what we don't want to see. On the other hand, the target DC voltage can't achieve with considering the different current limiting modes. 
In this paper, considering the different current limiting modes, a shutdown strategy of converter station is proposed, which can maximize the energy of each station back to the grid, to further improve the utilization of energy.

\section{Modeling of MMC}

Three-phase modular multi-level inverter topology is shown in Figure 1. Each arm consists of N sub-modules (SM) and an arm reactor in series, wherein each SM consists of two IGBT (VT1 and VT2) and the corresponding freewheeling diodes (VD1 and VD2), a DC side capacitor and a clamp resistors [3] [4].

When the bridge arm circulation is ignored, can be obtained [5]:

$$
\left\{\begin{array}{l}
u_{p a}=\frac{U_{d c}}{2}-u_{c a} \\
u_{n a}=\frac{U_{d c}}{2}+u_{c a}
\end{array}\right.
$$

Equation (1) shows that a desired AC output voltage can be obtained by controlling the voltages of the upper and lower bridge arms. To make the stability of the current system, there must be one side of the converter stations using fixed DC voltage controller.

\section{Control Strategy for Energy Feedback}

As the literature [2] says, entering discharge phase after the actual DC voltage dropping to $1.414 U_{l}$ can reach the maximum of energy feedback ratio in theory.

Considering the overload capacity of inverter itself, a current limiting control for an inverter is necessary. The current limiting methods must determine according to the practical application, which are the following three [6]:

a) Current limiting mode one: When one party of active current and reactive current reaches the limit, both decreases in the same proportion.

b) Current limiting mode two: reactive power is transferred prior to active current, which allows to a greater reactive power output at the cost of reducing active power, in order to achieve a stable system voltage.

c) Current limiting mode three: active power is transferred prior to reactive current, which allows to a larger active power output at the expense of reducing reactive power, in order to achieve important load power supply.

Energy feedback process is essentially a constantly adjusting the DC voltage drop process. When current limiting mode three is used, there must be one side of the converter stations using fixed DC voltage controller to drop the DC voltage accurately and effectively, and for this side, the converter station connected to active grid is selected generally. For other limitation modes, the stop process allows the inverter to absorb some of the inductive reactive.

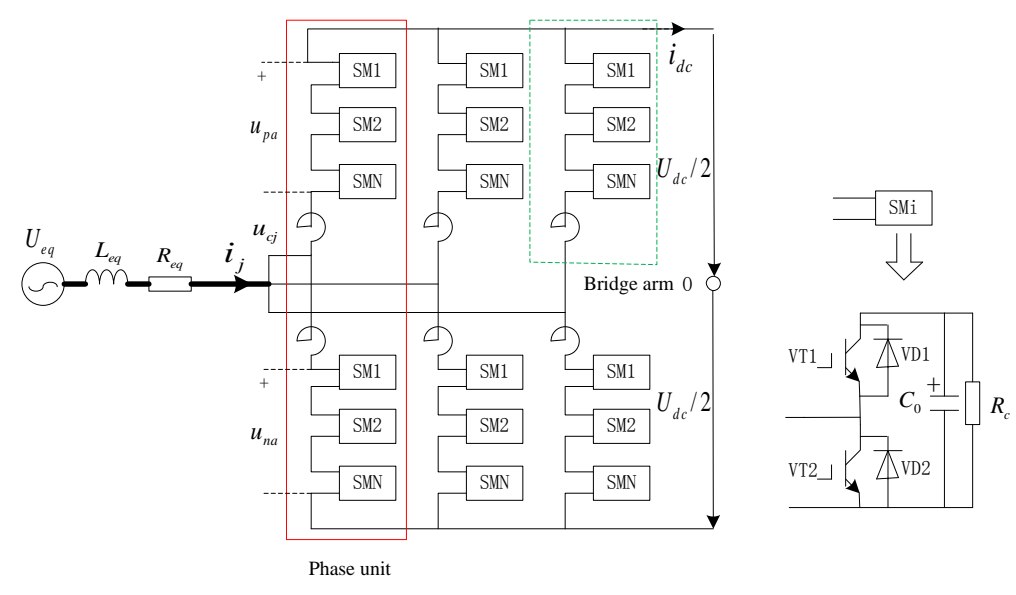

Figure 1. Topologies of a three-phase MMC and a sub-module. 
According to two-stage starting process for the converter station, the DC voltage is starting to lift from the AC line voltage peak after unlocking the converter station, and capacitor charging power is active power which is used to continues to charge the sub-module capacitors in the process of lifting the DC voltage, so the ultimate goal of energy feedback should be returned to the end state of that in which the charging current is out of control.

Figure 2 shows the structure of the inner current controller for inverter station. Figure $\mathbf{3}$ shows its quivalent circuit diagram.

Where: $u_{c d r e f} u_{c q r e f}$ respectively is the reference d, q-axis component of voltage modulation wave; $u_{s}$ is the system AC inistantaneous voltage; $u_{c}$ is the instantaneous voltage at the AC outlet of the inverter.

According to the equivalent circuit, in terms of an example for $\mathrm{AB}$ phase, can be obtained:

$$
\begin{aligned}
& u_{c a}-u_{c b}=u_{s a}-u_{s b}-i * 2 R-2 L \frac{d i}{d t} \\
& u_{c j}=u_{n j}-\frac{1}{2} U_{d c_{-} e q}=\operatorname{round}\left(\frac{u_{c j r e f}}{U_{S M}}\right) * U_{S M} \leq \frac{1}{2} U_{d c_{-} e q}
\end{aligned}
$$

where: $n_{\text {down }}=\frac{N}{2}+\operatorname{round}\left(\frac{u_{\text {cref }}}{U_{S M}}\right) \leq N$.

When current limiting mode three is used, the relationship $u_{c q r e f}=\sqrt{1-u_{c d r e f}}($ p.u. $)$ is established. At this time, the above goal cannot achieve, the DC voltage only can be reduced to a minimum

$U_{\mathrm{dc}}=2 u_{\text {cref }}=2 \sqrt{u_{\text {cdref }}^{2}+u_{\text {crref }}^{2}}=2 \sqrt{2} U_{s}=1.633 U_{l}$, where: $U_{s}$ is the system phase voltage fundamental RMS; $U_{l}$ is the system line voltage fundamental RMS.

When the converter station uses other current limiting modes, the auxiliary trigger strategy can be used to decline sub-module capacitor voltage further, at this time, the reference DC voltage is $1.414 U_{l}$. For this auxiliary trigger strategy, when the arm current make capacitors discharge, the sub-modules are put into until the capacitor voltages meet the requirements. But in the DC voltage reduction process, MMC also absorbs some of the inductive reactive power from the AC system.

\subsection{Both Sides of the Converter Stations Are Connected to an Active Network}

When both sides of the converter stations are connected to an active network, the two stations are only allowed to operate the switches after lockout. When current limiting mode three is used, the ultimate goal of energy feedback for the two stations is that voltages of the sub-module capacitors drop to $1.633 U_{l} / N$.

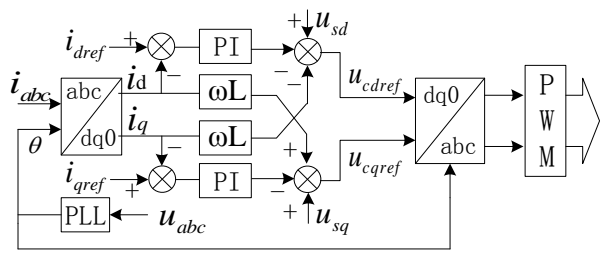

Figure 2. Structure of the inner current controller for inverter station.

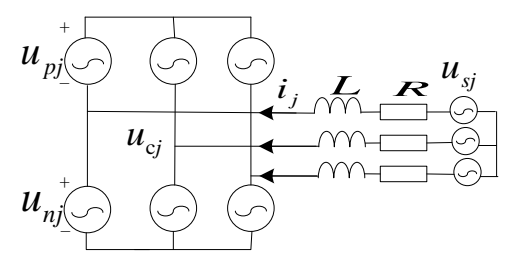

Figure 3. Equivalent circuit diagram for inverter station. 
$\delta$ is defined as the ratio of the energy feedback to the grid representing the sub-module initial stored energy, then

$$
\delta=\frac{U_{c_{-} r e f}^{2}-U_{c_{-} \min }^{2}}{U_{c_{-} r e f}^{2}}
$$

where: $U_{c_{-} r e f}$ is sub-modules rated capacitor voltage; $U_{c_{-} \min }$ is sub-module capacitor voltage in the end of energy feedback stage.

\subsection{Converter Station Supplies Power to Passive Network}

When the converter station supplies power to passive network, the AC load line can be disconnected after receiving the stop command, then the passive side of the converter station may contribute energy to the grid further compared to the former situation. Outage process is on contrary of startup procedure for a converter station to some degree. So the total input module number each phase should also be $2 \mathrm{~N}$ in the end of the energy feedback process.

A low-voltage discharge unlocking strategy is proposed, which selects a higher voltage sub-module capacitor to discharge in the moment of changing level, for that VT1 is conducted and VT2 is off, the rest of the submodules are removed. To speed up capacitor discharge process in the passive side, the inverter side is lowvoltage unlocked before dropping DC voltage in the rectifier side. In addition, to prevent a reverse DC current because of the difference between equivalent DC voltages at both ends of the DC line in the early energy feedback stage, the input module number each phase is gradually increased from $\mathrm{N}$ to $2 \mathrm{~N}$.

\section{Control Strategy for Energy Consumption}

There are a great many sub-modules in series in actual MMC projects, discharge resistor is access to constitute sub-module discharge circuit to accelerate the sub-module discharge, for which access locations are shown in Figure 4.

After the controller receives the transmitted energy discharge command, first latch the trigger pulse of all submodules, then cut off AC breaker, then cut off the DC breaker, and finally access to discharge resistor Rd to the DC side.

In this paper, each sub-module unit discharges in turn: First, for the sub-module SM1, VT1 is conducted, VT2 is off, and for the rest of the modules, VT1 and VT2 are both conducted, so that only one module is put into the discharge loop at the same time. When the SM1 capacitor voltage is reduced to meet the requirement, the SM2 capacitor turns to put into the discharge circuit, and so on. After the discharge stage for all modules each phase ends, trigger pulses of all modules are lockout.

In addition, there are three-phase bridge arms discharging in the same time and three-phase arms discharging in order. In this paper, three-phase bridge arms are discharged in the same time. For this, there is an interaction in inhibiting discharge phenomenon between three-phase bridge arms, which is mainly caused by the unbalance

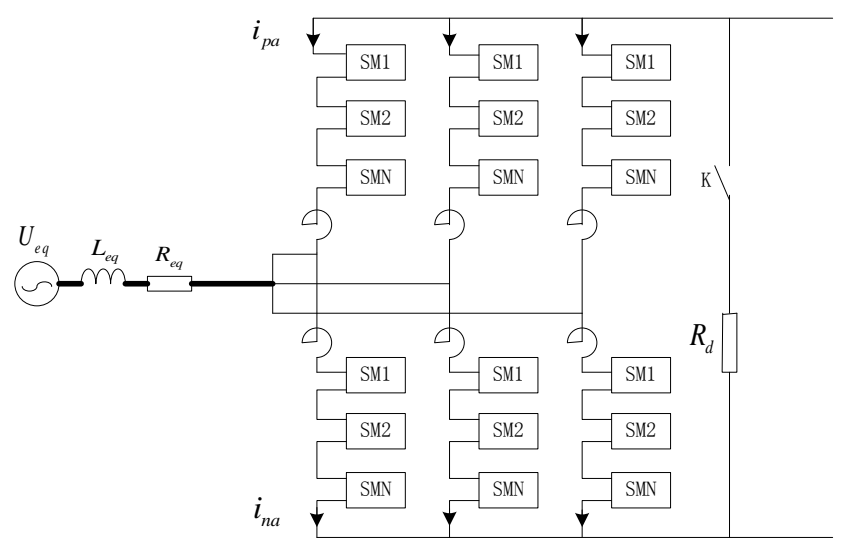

Figure 4. Discharge resistor access position. 
of sub-module capacitor voltage in three-phase bridge arms. This inhibited discharge between three-phase bridge arms will extend the total discharge time for converter stations to some extent.

\section{Simulation}

The simulation model is established in the MATLAB, and the parameters of the system are shown in Table $\mathbf{1}$.

The simulation time is set as six seconds, and the step size is $2.5 \mathrm{e}-6 \mathrm{~s}$.

\subsection{Waveforms from Simulation 1}

In this section, the shutdown process when both sides of the converter stations are connected to an active network is simulated. Figure 5 shows the DC voltage during this shutdown process, while DC reference voltage varies to $271 \mathrm{kV}$ at $3.2 \mathrm{~s}$; Figure 6 shows 20 sub-module capacitor voltages in a phase bridge arm; Figure 7, Figure 8 shows the active power and reactive power absorbed in both sides of converter stations.

Through analysis of the above waveforms, the following conclusions can be obtained:

1) Simulation results verify that the DC voltage can only drop to $1.633 \mathrm{U}_{1}$ by adjusting the fixed DC voltage

Table 1. Parameters used for simulation.

\begin{tabular}{cc}
\hline DC bus voltage & $320 \mathrm{kV}$ \\
Rated line-line voltage & $166 \mathrm{kV}$ \\
Number of module in one arm & 20 \\
Module capacitor & $50 \mathrm{mF}$ \\
Rated module voltage & $16 \mathrm{kV}$ \\
Arm inductor & $100 \mathrm{mH}$ \\
Cell clamping resistor & $4 \mathrm{e}^{10} \Omega$ \\
Discharge resistance & $20 \Omega$ \\
\hline
\end{tabular}

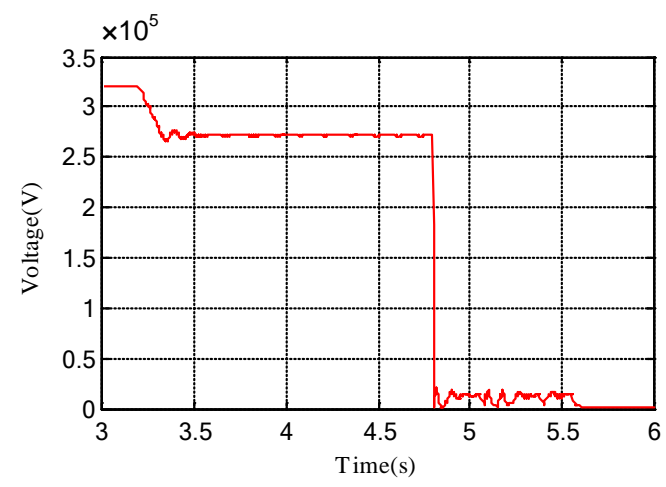

Figure 5. DC voltage.

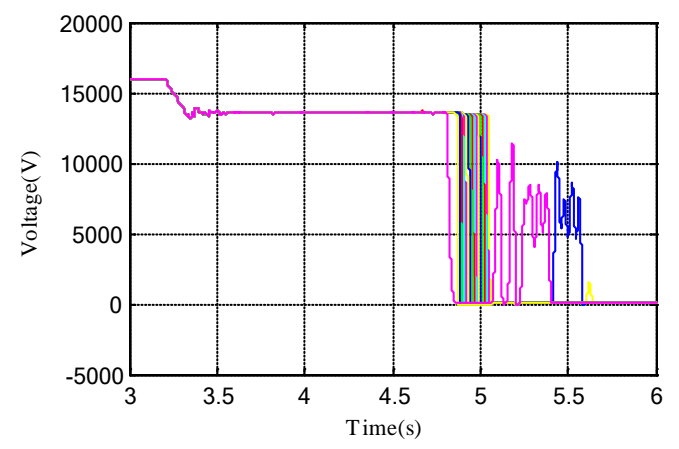

Figure 6. Sub-module capacitor voltages in a bridge arm. 

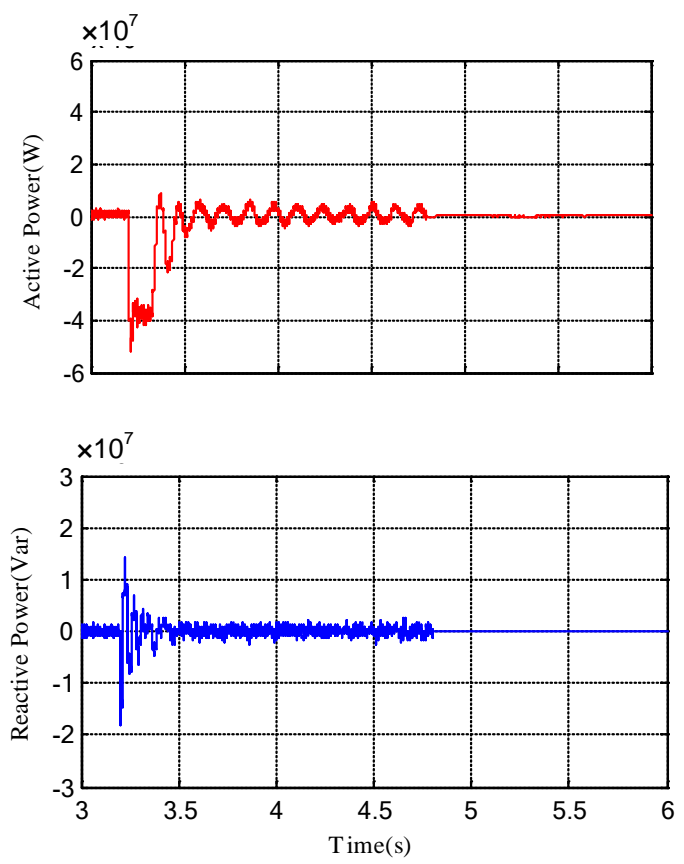

Figure 7. Power absorbed in the given DC voltage side.
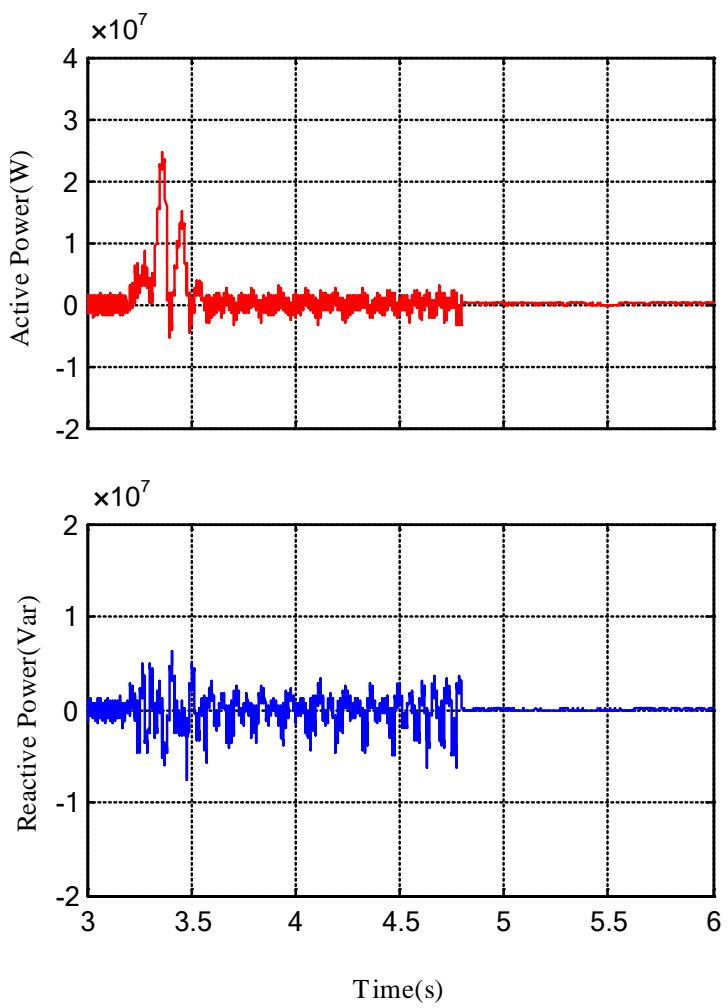

Figure 8. Power absorbed in the given PQ side.

controller with using the current limitation mode three.

2) The distribution of energy can be seen from power waveforms at both ends of the converter station. In the entire energy feedback process, capacitive stored energy and some energy absorbed form the AC grid connected to the given PQ side are passed to the AC grid connected to the given DC voltage side. 


\subsection{Waveforms from Simulation 2}

In this section, the shutdown process when the converter station supplies power to the passive network is simulated. Figure 9 shows the DC voltage during the shutdown process, while disconnect AC breaker in the passive side at $1.8 \mathrm{~s}$ and DC reference voltage varies to $271 \mathrm{kV}$ at $1.84 \mathrm{~s}$; Figure 10 and Figure 11 show 20 sub-module capacitor voltages in a phase bridge arm in both sides of converter stations; Figure 12 shows the active power and reactive power absorbed in the active side.

Through the above analysis of the waveform, the following conclusions can be obtained:

1) In the process of dropping the DC voltage by the fixed DC voltage controller, increasing the input submodule number per phase gradually cannot cause large DC current due to the surge of equivalent DC voltage in the passive side.

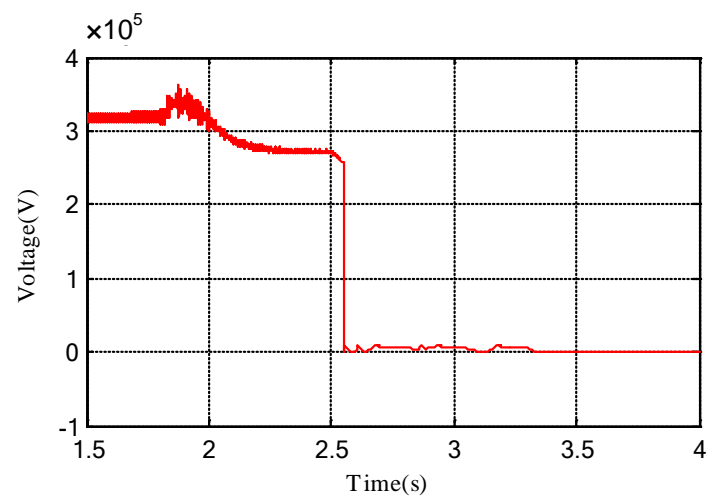

Figure 9. DC voltage.

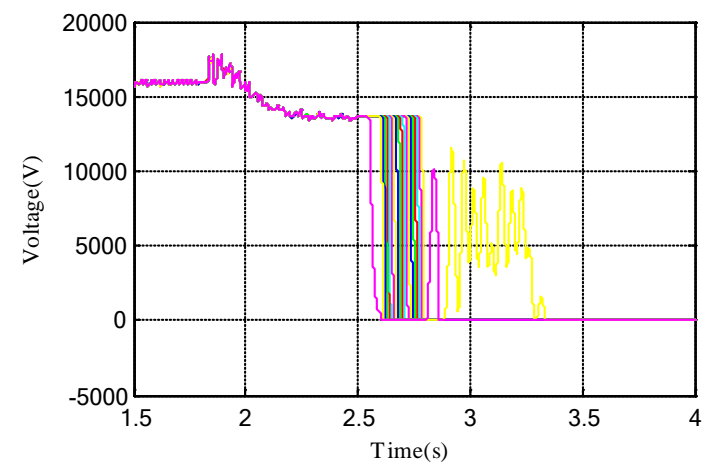

Figure 10. Sub-module capacitor voltages in a bridge arm in the active side.

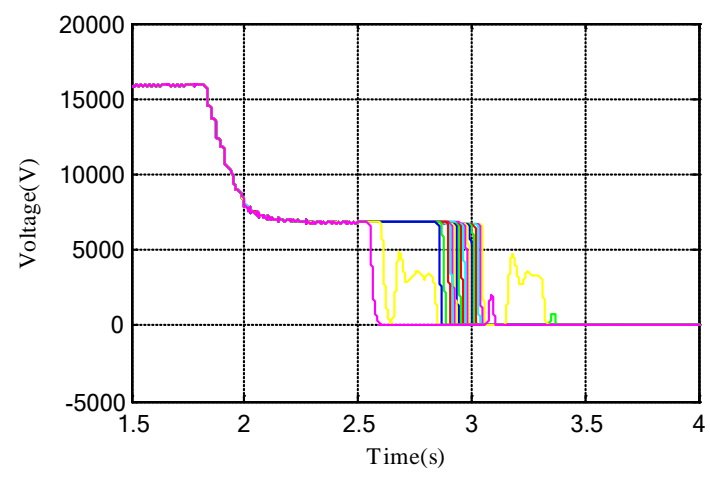

Figure 11. Sub-module capacitor voltages in a bridge arm in the passive side. 

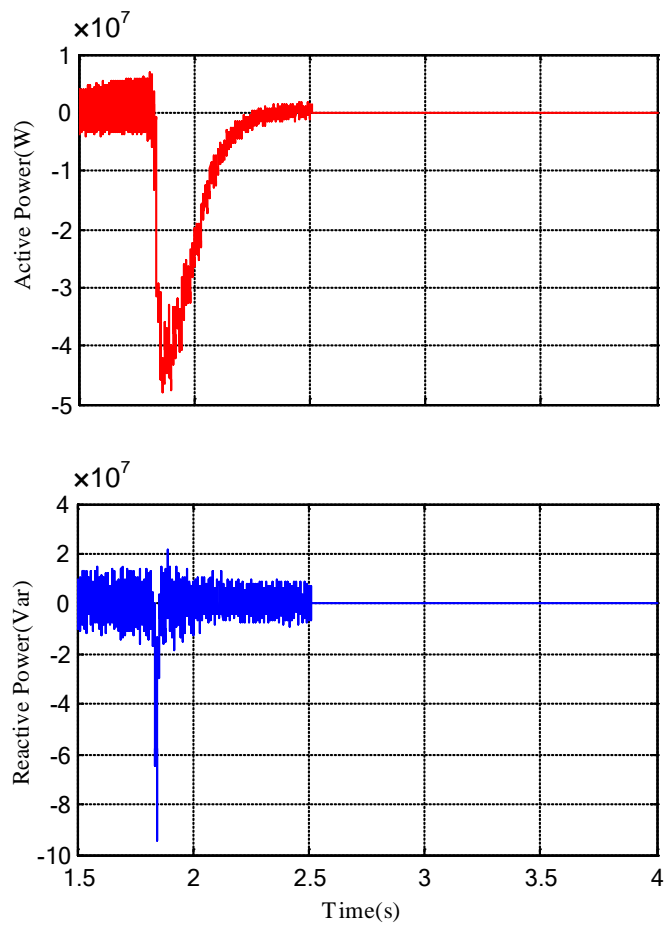

Figure 12. Power absorbed in the active side.

2) Throughout the energy feedback process, the energy stored in sub-module capacitances in the passive side is transferred to the AC power grid connected to the given DC voltage side along with that in the active side, and the output of inductive reactive power is up to about $10 \mathrm{M}$ Var.

3) Although three-phase bridge arms are discharged in the same time, but if two-phase capacitor voltages are not equal, B (A) bridge arm capacitors will charge A (B) phase capacitors again after a certain amount of discharge. On the other hand, the highest capacitor voltage phase will suppress the remaining two phases to discharge.

\section{Conclusion}

Energy feedback process can be seen as an inverse process of the controlled sub-stage in the pre-charge stage for MMC-HVDC, so the pre-charge control strategy can be migrated to the stop process with slight changes. Stop strategies for different current limitation modes are proposed, which can feed the maximum of the energy stored in the capacitors back to the grid. In addition, energy consumption resistance pressure and power levels are relatively low, which helps to reduce engineering costs and improve economic efficiency.

\section{Acknowledgements}

The project is supported by National High Technology Research and Development Program, Technology Research and Development of China 863 Program (2011AA05A102).

\section{References}

[1] Zhou, Y.B., Jiang, D.Z., Guo, J., et al. (2013) The Start and Stop Control for Modular Multi-Level Inverter Based HVDC Transmission System. Grid Technology, 36, 204-208.

[2] Song, P.G., Li, Y.F., Wang, L.N., Jiang, L. and Duan, C.T. (2013) Stop Strategy for MMC-HVDC Connected to Passive Network. Grid Technology, 11, 3247-3253.

[3] Dorn, J., Huang, H. and Retzmann, D. (2008) A New Voltage-Sourced Converter Topology for HVDC Applications. Cigré Session, B4-304, Paris.

[4] Xu, Z., et al. (2013) Voltage Source Converter Based High Voltage DC Transmission Systems. Mechanical Industry 
Press, Beijing.

[5] Guan, M.Y. and Xu, Z. (2010) The Modeling and Control of Modular Multilevel Converter Based HVDC Transmission System. Power System Automation, 19, 64-68.

[6] Tang, G.F. (2010) Voltage Source Converter Based High Voltage DC Transmission Systems. China Electric Power Press, Beijing. 